\title{
Computation of bounds for anchor problems in limit analysis and decomposition techniques
}

\author{
J J Muñoz, N Rabiei, A Lyamin, and A Huerta
}

\begin{abstract}
Numerical techniques for the computation of strict bounds in limit analyses have been developed for more than thirty years. The efficiency of these techniques have been substantially improved in the last ten years, and have been successfully applied to academic problems, foundations and excavations. We here extend the theoretical background to problems with anchors, interface conditions, and joints. Those extensions are relevant for the analysis of retaining and anchored walls, which we study in this work. The analysis of three-dimensional domains remains as yet very scarce. From the computational standpoint, the memory requirements and CPU time are exceedingly prohibitive when mesh adaptivity is employed. For this reason, we also present here the application of decomposition techniques to the optimisation problem of limit analysis. We discuss the performance of different methodologies adopted in the literature for general optimisation problems, such as primal and dual decomposition, and suggest some strategies that are suitable for the parallelisation of large three-dimensional problems. The propo sed decomposition techniques are tested against representative problems.
\end{abstract}

J J Munoz

Universitat Politècnica de Catalunya (UPC), Laboratori de Càlcul Numèric (LaCàN), Barcelona, Spain e-mail: j.munoz@upc.edu

N Rabiei

Universitat Politècnica de Catalunya (UPC), Laboratori de Càlcul Numèric (LaCàN), Barcelona, Spain e-mail: nima.rabiei@upc.edu

A Lyamin

University of Newcastle, Center for Geothecnical and Materials Modelling, Newcastle, Australia e-mail: a.lyamin@newcastle.edu.au

A Huerta

Universitat Politècnica de Catalunya (UPC), Laboratori de Càlcul Numèric (LaCàN), e-mail: antonio.huerta@upc.edu 


\section{Introduction}

\subsection{Limit analysis problem}

According to the lower (primal) and upper (dual) bound theorem of limit analysis, the bearing capacity of a structure is equal to $(i)$ the maximum load factor $\lambda^{*}$ under equilibrium conditions and with plastically admissible stresses $\sigma$, (i.e. they belong to a set $\mathscr{B})$, or alternatively, to $(i i)$ the minimum dissipation energy $D(v)$ of a kinematically admissible velocity field $v$. Mathematically, the resulting bearing capacity has the structure of a saddle point problem that can be written as [6],

$$
\lambda^{*}=\min _{\ell(v)=1} \max _{\substack{\sigma \in \mathscr{B} \\ a(\sigma, v)=\lambda \ell(v)}} a(\sigma, v)
$$

where the linear form $\ell(v)$ is the power dissipated by the external loads, while the bilinear form $a(\sigma, v)$ is the internal dissipated power. For a given domain $\Omega$, subjected to external surface load $g$ and body load $f$, these forms are explicitly given by:

$$
\begin{aligned}
\ell(v) & =\int_{\Omega} f \cdot v d \Omega+\int_{\partial \Omega} g \cdot v d \Gamma \\
a(\sigma, v) & =\int_{\Omega} \sigma: \varepsilon(v) d \Omega+\int_{\Gamma} \sigma:(\llbracket v \rrbracket \bar{\otimes} n) d \Gamma
\end{aligned}
$$

with $\Gamma$ the (unknown) region of $\Omega$ where the velocity is discontinuous, $\llbracket v \rrbracket$ the velocity discontinuity, and $n$ the normal vector at this discontinuity. The operator $\bar{\otimes}$ is the symmetrised dyadic product such that $a \bar{\otimes} b=\frac{1}{2}(a \otimes b+b \otimes a)$ (see also [10] for equivalent definitions of discontinuous velocities). The saddle point problem or min max problem can be also rewritten as,

$$
\begin{aligned}
\lambda^{*} & =\max _{\substack{\sigma \in \mathscr{B} \\
a(\sigma, v)=\lambda \ell(v), \forall v}} \lambda \\
& =\min _{\ell(v)=1} D(v)
\end{aligned}
$$

where the dissipated energy $D(v)$ is defined by,

$$
D(v)=\max _{\substack{\sigma \in \mathscr{B} \\ a(\sigma, v)=\lambda \ell(v)}} a(\sigma, v)
$$

Equations (2) and (3) are the primal and dual form of the saddle point problem in (1). From the dual form in (3), it turns out that the velocities must be associated, that is, that $\varepsilon(v) \in \partial \mathscr{B}$ and $\llbracket v \rrbracket \otimes n \in \partial \mathscr{B}$, with $\partial \mathscr{B}$ the sub-gradient of set $\mathscr{B}$.

The analytical saddle point problem in (1) is illustrated in Figure 1a. A stress field such that $\sigma \in \mathscr{B}$ and for which the equilibrium condition $a(\sigma, v)=\lambda \ell(v), \forall \ell(v)$ is satisfied everywhere is so-called a statically admissible stress space. Similarly, 
a velocity field such that $\ell(v)=1$ and for which the associative conditions $\llbracket v \rrbracket \otimes$ $n, \varepsilon(v) \in \partial \mathscr{B}$ is satisfied everywhere is so-called a kinematically admissible space. The saddle point problem in (1) states that the bearing capacity (or maximum load factor) of a structure is equal to the internal dissipated energy of a statically admissible stress space $\sigma^{*}$ and a kinematically admissible velocity space $v^{*}$.

\subsection{Discrete upper and lower bound formulations}

By choosing an appropriate discrete statically admissible space $\left(\sigma^{L B}, v^{L B}\right)$ that satisfies the maximisation conditions in (1), and a kinematically admissible space $\left(\sigma^{U B}, v^{U B}\right)$ that satisfies the minimisation conditions in (1), we can construct strict bounds of the load factor $\lambda^{*}$ as,

$$
\lambda^{L B}=a\left(\sigma^{L B}, v^{L B}\right) \leq \lambda^{*} \leq a\left(\sigma^{U B}, v^{U B}\right)=\lambda^{U B}
$$

A schematic of discrete spaces $\left(\sigma^{L B}, v^{L B}\right)$ and $\left(\sigma^{L B}, v^{L B}\right)$ that satisfy those conditions are given in Figure 2. The reader is referred to $[13,14]$ for a justification of those spaces. When resorting to them, the saddle point problem is consequently modified, as illustrated in Figures 1b-c. The max min problem in (1) turns into a lower bound problem when the first pair of spaces is used, and into an upper bound problem when the second pair is used.

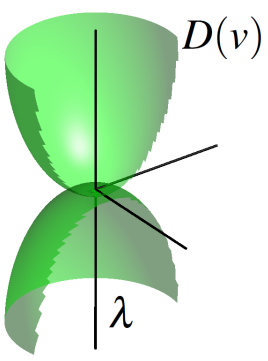

(a)

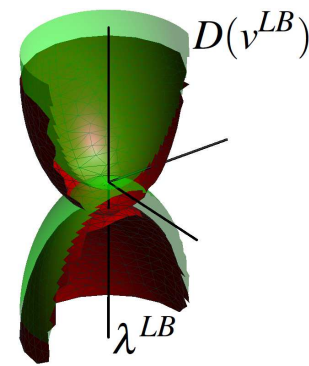

(b)

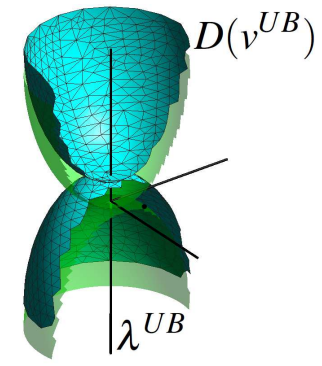

(c)

Fig. 1 Illustration of saddle point problem for the (a) analytical solution of limit analysis problem, (b) lower bound discrete limit analysis problem, and (c) upper bound discrete limit analysis problem.

Therefore, after using these interpolation spaces $\left(\sigma^{U B}, v^{U B}\right)$ and $\left(\sigma^{L B}, v^{L B}\right)$ in the exact optimisation problem in (2) or (3), we are able to compute exact upper and lower bounds of the optimal factor $\lambda^{*}$. In particular, the lower bound problem turns into the following form, 


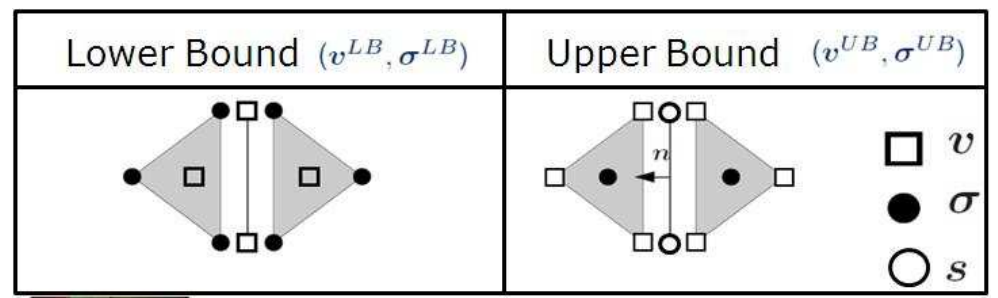

Fig. 2 Interpolation spaces for the lower and upper bound problem. Symbols at nodes denote a elementwise linear space, while symbols at the center of the element denote elementwise constant space.

$$
\begin{aligned}
\lambda^{L B}= & \max _{\sigma^{U B}} \lambda \\
\text { s.t. } & \left\{\begin{array}{l}
\overline{\mathbf{A}}^{e q 1} \sigma^{L B}+\lambda F^{e q 1}=\mathbf{0} \\
\overline{\mathbf{A}}^{e q 2} \sigma^{L B} \\
\overline{\mathbf{A}}^{e q 3} \sigma^{L B}+\lambda F^{e q 3}=\mathbf{0} \\
\sigma_{i}^{L B, e} \in \mathscr{B},
\end{array}\right.
\end{aligned}
$$

whereas the upper bound problem reads,

$$
\begin{aligned}
\lambda^{U B}= & \min _{v^{U B}} D\left(v^{U B}\right) \\
\text { s.t. } & \left\{\begin{array}{l}
l\left(v^{U B}\right)=1 \\
-\varepsilon\left(v_{i}^{U B, e}\right) \in \partial \mathscr{B}^{*}, \quad e=1, \ldots, N_{e}, i=1, \ldots, n_{s d}+1 \\
-\llbracket v^{U B} \rrbracket_{j}^{\xi} \bar{\otimes} n^{\xi} \in \partial \mathscr{B}^{*}, \xi=1, \ldots, N_{\xi}, j=1, \ldots, n_{s d}
\end{array}\right.
\end{aligned}
$$

where $n_{s d}$ is the number of space dimensions, and the three block equations in (4) correspond respectively to the intra-element equilibrium, the inter-element equilibrium, and the Neumann boundary conditions. The vectors $\sigma^{L B}$ and $v^{U B}$ contain the collection of all nodal stresses $\sigma_{i}^{L B, e}$ of element $e$, and the velocities $v_{i}^{U B, e}$ and $v_{j}^{U B, \xi}$ for each node $i$ of element $e$ or each node $j$ of edge $\xi$, respectively.

The problems above can be solved efficiently using available optimisation programs [17, 18, 1]. Moreover, for the usual plasticity criteria such as von Mises or Mohr-Coulomb in two dimensions, we can apply linear transformations of the stress variables that turn the membership conditions $\sigma \in \mathscr{B}$ into second order cones (SOC), which can be handled by the mentioned optimisation software. After apply in such transformations, the optimisation problems above turn into,

$$
\begin{aligned}
\lambda^{L B}= & \max _{x} \lambda \\
\text { s.t. } & \left\{\begin{array}{l}
\mathbf{A}^{e q 1} x+\lambda F^{e q 1}=b^{e q 1} \\
\mathbf{A}^{e q 2} x \quad=\mathbf{0} \\
\mathbf{A}^{e q 3} x+\lambda F^{e q 3}=b^{e q 3} \\
x_{i}^{L B, e} \in \mathscr{K}, \quad e=1, \ldots, N_{e}, i=1, \ldots, n_{s d}+1
\end{array}\right.
\end{aligned}
$$




$$
\begin{aligned}
\lambda^{U B}= & \min _{v^{U B}} b \cdot v^{U B} \\
\text { s.t. } & \begin{cases}l\left(v^{U B}=1\right. \\
v_{i}^{U B, e} \in \mathscr{K}^{*}, & e=1, \ldots, N_{e}, i=1, \ldots, n_{s d}+1 \\
\llbracket v^{U B} \rrbracket_{i}^{\xi} \bar{\otimes} n^{\xi} \in \mathscr{K}^{*}, \xi=1, \ldots, N_{\xi}, i=1, \ldots, n_{s d}\end{cases}
\end{aligned}
$$

with $\mathscr{K}$ a second order cone, and $\mathscr{K}^{*}$ the dual cone of $\mathscr{K}$ [4].

\subsection{Mesh adaptivity}

The optimum values of the lower and upper bound problem can be used to compute a set of elemental and edge contributions to the total gap, which are defined by $[7,14]$ :

$$
\begin{aligned}
& \Delta \lambda^{e}=\int_{\Omega^{e}} \sigma^{U B}: \varepsilon\left(v^{U B}\right) d \Omega+\int_{\Omega^{e}} \nabla \cdot \sigma^{L B} \cdot v^{U B} d \Omega-\int_{\partial \Omega} \sigma^{L B} n \cdot v^{U B} d \Gamma \\
& \Delta \lambda^{\xi}=\int_{\Gamma^{\xi}} \sigma^{U B, \xi} \cdot \llbracket v^{U B} \rrbracket d \Gamma-\int_{\Gamma^{\xi}} \sigma^{L B} n \cdot \llbracket v^{U B} \rrbracket d \Gamma
\end{aligned}
$$

These bound gaps satisfy the properties, $\lambda^{U B}-\lambda^{L B}=\sum_{e} \Delta \lambda^{e}+\sum_{\xi} \Delta \lambda^{\xi}, \Delta \lambda^{e} \geq$ 0 and $\Delta \lambda \xi \geq 0$, which make them good candidates to estimate the errors of the lower and upper bound solution. These quadratures are obtained only if appropiate quadratures are employed to compute the integrals: Gauss quadrature for all the terms excepting the first integral in $\Delta \lambda \xi$ (see [15] for the justification of this). We have used them to design an adaptive remeshing strategy employed in Section 4.1.

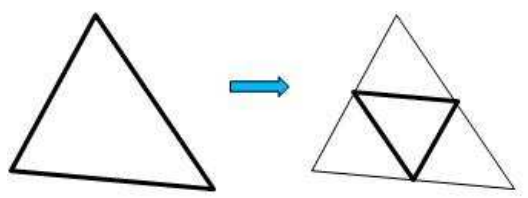

(a)

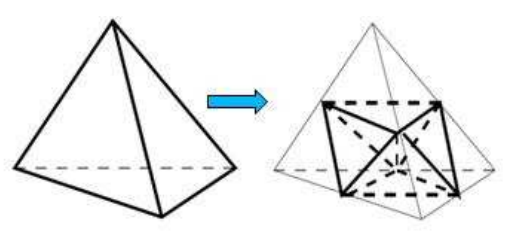

(b)

Fig. 3 Subdivision of a triangular element in 2D (a) and tetrahedron in 3D (b), when their elemental gap contribution $\Delta \lambda^{a}$ is larger than a pre-defined thereshold. 


\section{Extension to anchors, joints and frictional interfaces}

We will develop next specific conditions for common interface conditions encountered in geomechanics. In all cases we add specific constraints that preserve the strictness of the bounds. The studied and implemented situations are:

1. Interface material that splits two different materials with specific admissibility criterion for the common boundary.

2. Duplicated edges: in two-dimensional applications, it may convenient to overlap materials or structural elements such as ties or anchors. In these situations, it is required to have edges that joint one element on one side and two elements, $B$ and $B^{\prime}$, on the other side.

3. Modelling of joints such as articulated joints in anchors and anchor-wall interface.

We briefly describe how to include in each case the corresponding constraints in the optimisation problem (see [16] for further details).

\subsection{Interface conditions}

Specific admissibility conditions for the stresses can be introduced by adding new nodal variables $\sigma_{i}^{I}$ at the interface, with new membership sets $\mathscr{B}_{I}$, that are different from the two materials at each side of the interface. These new nodal variables are equivalent to extending the spaces given in Section 1.2, and adding equilibrium constraints such as,

$$
\begin{aligned}
\left(\sigma_{i}^{A}-\sigma_{i}^{B}\right) \cdot n=\mathbf{0}, & i=1,2 \\
\left(\sigma_{i}^{A}-\sigma_{i}^{I}\right) \cdot n=\mathbf{0}, & i=1,2
\end{aligned}
$$

and adding the following membership constraints at the interface,

$$
\sigma_{i}^{I} \in \mathscr{B}_{I}, \quad i=1,2
$$

The vector $n$ is the normal to the interface edge or face in 3D. Some of admissible sets that may be employed in common problems are depicted in Figure 4. We also note that the nodal velocities at the two edges between $A$ and $B$, indicated in Figure $4 \mathrm{a}$ with circles, correspond in fact to the Lagrange multipliers associated with these constraints: the velocities at one edge are associated with equation (7) while those at the other edge correspond to equation (8).

Figure 5 shows the usual admissible domain $\mathscr{B}_{I}$ for the stresses at the interfaces. The criteria in Figure $5 \mathrm{c}$ has been included for completeness reasons, but it is unrealistic and has not practical interest. The subscript $\sigma_{N}$ and $\sigma_{T}$ denote the normal and tangential components of the traction vector $\sigma \cdot n$ at the interface. In our imple- 
mentation, we impose admissibility domains for the stress tensors $\sigma^{I}$, which imply the usual admissibility conditions for the traction vectors $\sigma^{I} \cdot n$.

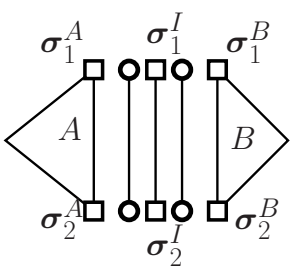

(a)

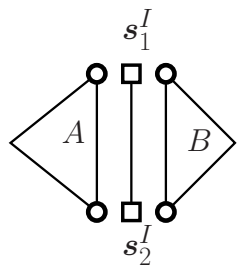

(b)

Fig. 4 Discrete spaces considered for the lower (a) and upper (b) bound problems when modelling interfaces.

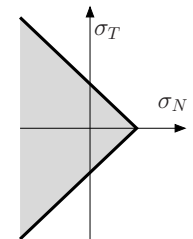

(a)

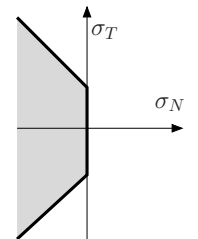

(b)

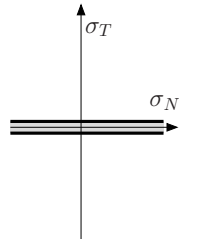

(c)

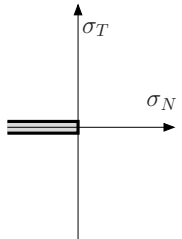

(d)

Fig. 5 Interface conditions: Rough interface, equal to soil properties (a), Rough with no tension (b), smooth interface (c), and smooth with no tension (d).

\subsection{Duplicated edges}

The modelling of two-dimensional problems with anchors, ties or reinforcements can be achieved by superimposing the latter elements onto the soil elements. This is an idealisation of the real three-dimensional situation. We consider the twodimensional plane strain analysis with additional superimposed elements (reinforcements, ties or anchors) that are analysed in plane-stress and connected to the soil. We have modelled the structural elements as solid elements and not linear elements, which prevents the presence of point loads and therefore allows us to preserve the strictness of the bounds.

Computationally, we need to deal with edges where the soil elements on side $A$ are connected to two types of elements: other soil elements (with variables $B$ ) and those superimposed elements that model the structural elements (with variables $B^{\prime}$ ). Figure 6 shows such an edge in the lower and upper bound formulation. We will call those edges as duplicated edges.

In the lower bound formulation, we need to modify the equilibrium constraints of the edges, which now read, 


$$
\left(\sigma_{i}^{A}-\sigma_{i}^{B}-\sigma_{i}^{B^{\prime}}\right) \cdot n=0, i=1,2
$$

where $\sigma_{i}^{A}, \sigma_{i}^{B}$ an $\sigma_{i}^{B^{\prime}}$ are respectively the nodal stresses at sides $A, B$ nd $B^{\prime}$. Since there is one equilibrium equation per common node, each duplicated edge requires two nodal velocities, as indicated with circles in Figure 6a.

In the upper bound formulation, the dissipation power at the edge corresponds to the sum of the power dissipated between elements $A$ and $B$, and the dissipated power between elements $A$ and $B^{\prime}$. Formally, the total dissipation power at the edge correspond to the sum of two integrals along the common edge:

$$
a_{\xi}(\sigma, v)=\int_{\Gamma^{\xi}} \sigma^{A-B} \cdot\left(v^{B}-v^{A}\right) d \Gamma+\int_{\Gamma^{\xi}} \sigma^{A-B^{\prime}} \cdot\left(v^{B^{\prime}}-v^{A}\right) d \Gamma
$$

This expression of the dissipated power is equivalent to extending the stress space at the edges with two superimposed linear stress spaces, $\sigma^{A-B}$ and $\sigma^{A-B^{\prime}}$, indicated by two pairs of squares in Figure $6 \mathrm{~b}$. By imposing that each one of the four nodal variables is admissible, that is:

$$
\left\{\begin{array}{l}
\sigma_{i}^{A-B} \in \mathscr{B}_{I} \\
\sigma_{i}^{A-B^{\prime}} \in \mathscr{B}_{I}
\end{array}, i=1,2\right.
$$

with $\mathscr{B}_{I}$ the admissible set for the interface conditions, the admissibility of the corresponding velocity jumps $v_{i}^{A}-v_{i}^{B}$ and $v_{i}^{A}-v_{i}^{B^{\prime}}$ is ensured, and therefore the strictness of the upper bound is guaranteed. The nodal velocities are indicated with circles in Figure 6b.

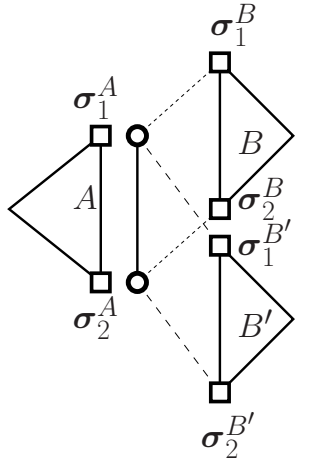

(a)

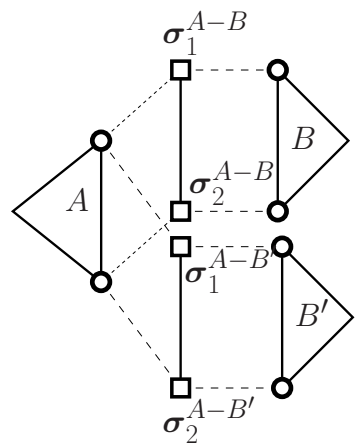

(b)

Fig. 6 Discrete spaces considered for the lower (a) and upper (b) bound problems when modelling duplicated edges. Elements $B$ and $B^{\prime}$ are geometrically superimposed. 


\subsection{Joints}

Some of the practical problems in limit analysis include anchors, ties or other structural elements that are connected through joints. In this case, the kinematic constraints must be included in the exact problem described in Section 1.1, and also modelled in such a way that the strictness of the bounds is preserved. In the lower bound formulation, the presence of joints is modelled by including solely a pointto-point equilibrium condition, that is,

$$
\int\left(\sigma^{A}-\sigma^{B}\right) \cdot n d \Gamma=\mathbf{0}
$$

which replaces the equilibrium along the whole edge between elements. In the upper bound problem, the construction of a kinematically admissible space for the velocities is constructed by constricting the relative displacements of the joints in such a manner that only rotations with respect to the joint centre are allowed. Formally, this is achieved by replacing the associative velocity field at the joint by the following constraint in the upper bound problem in (5):

$$
\llbracket v \rrbracket_{1}+\llbracket v \rrbracket_{2}=0
$$

Figure 7 shows a schematic of the stress and velocity spaces employed for the modelling of the joints in the lower and upper bound problem. We point out that the joint is without friction, since no dissipated energy is associated to the relative rotation at the joint. This is computational made explicit by not imposing any equilibrium relation associated to the rotations in equation (10), and imposing exactly the kinematic relation in (11), i.e. the associated Lagrangian multiplier (variable $s_{J}$ in Figure 7) is free.

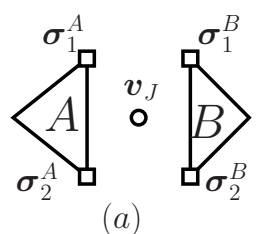

(a)

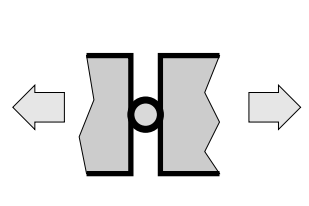

$v_{2}^{A} 8$

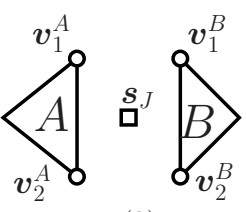

$(b)$

Fig. 7 Rotational joint and discrete spaces considered for the lower (a) and upper (b) bound problem.

\section{Decomposition techniques}

In order to reduce the memory requirement of realistic three dimensional problems we propose a decomposition of the optimization problems, which is based on the ideas explained in $[3,5]$. While the decomposition techniques for optimisation 
problems is a relatively recent topic, its application to limit analysis and other plasticity problems has found far less attention [12]. We also refer the reader to [11], where alternative decomposition techniques of the limit analysis problem has been introduced. We here first briefly describe some of the general ideals of decomposition of optimisation problems.

\subsection{Decomposition of optimisation problems}

\subsubsection{Primal Decomposition}

To illustrate the decomposition techniques, we state the following linear optimization problem:

$$
\begin{aligned}
& c^{T} x^{*}=\min _{x} c^{T} x \\
& \mathbf{A} x=b \\
& x \geq \mathbf{0},
\end{aligned}
$$

whose dual read,

$$
\begin{aligned}
& b^{T} y^{*}=\max _{y} b^{T} y \\
& \mathbf{A}^{T} y \leq c .
\end{aligned}
$$

The primal decomposition consists on rewriting the problem (12) as:

$$
\begin{gathered}
\min _{x_{1}, x_{2}} c_{1}^{T} x_{1}+c_{2}^{T} x_{2} \\
\mathbf{A}_{1} x_{1}+\mathbf{A}_{2} x_{2}=b \\
x_{1} \geq \mathbf{0}, x_{2} \geq \mathbf{0} .
\end{gathered}
$$

In other words, we split the primal $x$ variable as $x=\left(x_{1}, x_{2}\right)$. The problem above is equivalent to :

$$
\begin{aligned}
& \min _{t} \min _{x_{1}, x_{2}} c_{1}^{T} x_{1}+c_{2}^{T} x_{2} \\
& \mathbf{A}_{1} x_{1}=\frac{b}{2}+t \\
& \mathbf{A}_{2} x_{2}=\frac{b}{2}-t \\
& x_{1} \geq 0, x_{2} \geq 0, t \text { is free. }
\end{aligned}
$$

Given a fixed value of $t$, the optimum value may be obtained as a result of the inner minimums,

$$
\begin{aligned}
& f_{i}(t)=\min _{x_{i}} c_{i}^{T} x_{i} \\
& \mathbf{A}_{i} x_{i}=\frac{b}{2}+(-1)^{i+1} t \\
& x_{i} \geq \mathbf{0} \quad(i=1,2),
\end{aligned}
$$


so called sub-problems or slave problems and the following master problem:

$$
\min _{t} f_{1}(t)+f_{2}(t)
$$

which only depends on the global variable $t$. The Lagrangian function of problem (14) is given by:

$$
\begin{aligned}
L\left(x_{1}, x_{2}, y_{1}, y_{2}, w_{1}, w_{2}\right)= & c_{1}^{T} x_{1}+c_{2}^{T} x_{2}+y_{1}^{T}\left(\frac{b}{2}+t-\mathbf{A}_{1} x_{1}\right) \\
& +y_{2}^{T}\left(\frac{b}{2}-t-\mathbf{A}_{2} x_{2}\right)-\mathbf{w}_{1}^{T} x_{1}-\mathbf{w}_{2}^{T} x_{2} \\
= & c_{1}^{T} x_{1}+y_{1}^{T}\left(\frac{b}{2}-\mathbf{A}_{1} x_{1}\right)+c_{2}^{T} x_{2}+y_{2}^{T}\left(\frac{b}{2}-\mathbf{A}_{1} x_{1}\right) \\
& +t^{T}\left(y_{1}-y_{2}\right) \\
= & L_{1}\left(x_{1}, t ; y_{1}, w_{1}\right)+L_{2}\left(x_{2}, t ; y_{2}, w_{2}\right),
\end{aligned}
$$

with

$$
L_{i}\left(x_{i}, t ; y_{i}, w_{i}\right)=c_{i}^{T} x_{1}+y_{i}^{T}\left(\frac{b}{2}+(-1)^{i+1} t-\mathbf{A}_{1} x_{1}\right)-w_{i}^{T} x_{i},(i=1,2) .
$$

It then follows that we can rewrite the optimum primal objective $c^{T} x^{*}$ as,

$$
c^{T} x^{*}=c_{1}^{T} x_{1}^{*}+c_{2}^{T} x_{2}^{*}=\min _{t} \sum_{i=1}^{2} \min _{x_{i}} \max _{y_{i}, w_{i}} L_{i}\left(x_{i}, t ; y_{i}, w_{i}\right) .
$$

After observing the equation above, we have that $\nabla_{t} L=\left(y_{1}-y_{2}\right)$, and therefore we can update the master variables with the following descent method,

$$
t^{k+1}=t^{k}-\alpha^{k}\left(y_{1}^{k}-y_{2}^{k}\right)=t^{k}+\alpha^{k}\left(y_{2}^{k}-y_{1}^{k}\right),
$$

where $y_{1}$ and $y_{2}$ are the sub-gradient of functions $f_{1}$ and $f_{2}$ respectively. $\alpha^{k}$ is a step length that can be chosen in any of the standard ways [3].

\subsubsection{Dual Decomposition}

We recall the same problem in (13). Dual decomposition for this example is straightforward. We form the Lagrangian function as follows: 


$$
\begin{aligned}
L\left(x_{1}, x_{2}, y, w_{1}, w_{2}\right)= & c_{1}^{T} x_{1}+c_{2}^{T} x_{2}+y^{T}\left(\frac{b}{2}-\mathbf{A}_{1} x_{1}+\frac{b}{2}-\mathbf{A}_{2} x_{2}\right) \\
& -w_{1}^{T} x_{1}-w_{2}^{T} x_{2} \\
= & \left(c_{1}^{T} x_{1}+y^{T}\left(\frac{b}{2}-\mathbf{A}_{1} x_{1}\right)-w_{1}^{T} x_{1}\right) \\
& +\left(c_{2}^{T} x_{2}+y^{T}\left(\frac{b}{2}-\mathbf{A}_{2} x_{2}\right)-w_{2}^{T} x_{2}\right),
\end{aligned}
$$

so we can minimize over $x_{1}$ and $x_{2}$ separately given the dual variable $y$, to find $g(y)=g_{1}(y)+g_{2}(y)$ where $g(y)$ is given as,

$$
g(y)=\min _{x_{1}, x_{2}} L\left(x_{1}, x_{2}, y, w_{1}, w_{2}\right)=\min _{x_{1}, x_{2}} L_{1}\left(x_{1}, y, w_{1}\right)+L_{2}\left(x_{2}, y, w_{2}\right) .
$$

In order to find $g_{1}(y)$ and $g_{2}(y)$, respectively, we solve the following two subproblems:

$$
\begin{aligned}
& g_{1}(y)=\min _{x_{1} \geq 0} c_{1}^{T} x_{1}+y^{T}\left(\frac{b}{2}-\mathbf{A}_{1} x_{1}\right)=\min _{x_{1} \geq 0}\left(c_{1}^{T}-\mathbf{A}_{1}^{T} y\right) x_{1}+y^{T} \frac{b}{2}, \\
& g_{2}(y)=\min _{x_{2} \geq 0} c_{2}^{T} x_{2}+y^{T}\left(\frac{b}{2}-\mathbf{A}_{2} x_{2}\right)=\min _{x_{2} \geq 0}\left(c_{2}^{T}-\mathbf{A}_{2}^{T} y\right) x_{2}+y^{T} \frac{b}{2} .
\end{aligned}
$$

The master algorithm updates $y$ based on sub-gradient as follows:

$$
y=y+\beta\left(\frac{b}{2}-\mathbf{A}_{1} x_{1}+\frac{b}{2}-\mathbf{A}_{2} x_{2}\right)=y+\beta(b-\mathbf{A} x) .
$$

where $\beta$ is a step length that can be chosen in any of the standard ways [3].

\subsubsection{Bender's decomposition}

One of the main disadvantages of the previous decomposition is the update of the master variables. Since the minimised/maximised functions are non-smooth, the updated in (17) and (18) is not always optimal. For this reason, we have also studied the implementation of Bender's decomposition [2, 9]. The latter may be applied to the simple form of the linear problem in (14) as the solution of the following two sub-problems,

$$
\left.\begin{array}{c}
\min _{x_{i}} c_{i}^{T} x_{i} \\
\quad \mathbf{A}_{i} x_{i}=\frac{b}{2}+(-1)^{i+1} t \\
\quad x_{i} \geq 0,
\end{array}\right\} i=1,2
$$

and the solution of the following master problem: 


$$
\begin{aligned}
\min _{\alpha, t} \alpha_{1} & +\alpha_{2} \\
0 & \geq\left(\frac{b}{2}+(-1)^{i+1} t\right) \cdot y_{i}^{p}, p=1, \ldots, p_{k} \\
\alpha_{i} & \geq\left(\frac{b}{2}+(-1)^{i+1} t\right) \cdot y_{i}^{q}, q=1, \ldots, q_{k}
\end{aligned}
$$

The first and second sets of inequalities in (20) are respectively so-called feasibility cuts or optimality constraints, where $y_{i}^{p}$ and $y_{i}^{q}$ are the whole set optimal dual variables and extreme rays of the dual problem of (19) computed up to iteration $k=p_{k}+q_{k}$. Further details on the Bender's decomposition may be found in [8].

\subsection{Decomposition of limit analysis optimisation problem}

The decomposition techniques described in the Section 3 are here adapted to optimization problem encountered in limit analysis. When applying these ideas, it must be taken into account the particular structure of the lower and upper bound optimization problems. Indeed, the objective function is only formed by the load factor in the lower bound problem, the constraints correspond to the equilibrium constrains, as a function of the stress variables which belongs to a non-linear set. As explained in Section 1 the constraints become linear equations of variables that belong to second-order cones. In the sequel we apply the decomposition technique described to the lower bound (LB) problem in limit analysis.

\subsubsection{Decomposition of LB problem}

The decomposition of LB problem corresponds to splitting the nodal stress variables $\sigma$ into two sets $\sigma^{1}$ and $\sigma^{2}$, which in turn is also equivalent to splitting the variable $x$ into two variables $x_{1}$ and $x_{2}$. In this case, the constrains may be decomposed like in the primal technique, by using additional traction variables $t$ between domains, as depicted in Figure 13. In other words we split our domain in two subproblems with local variables $x_{i}$, and a master(global) variable $t$ that in the LB problem corresponds to the internal tractions between the sub-domains. Such variables may be seen as a (non-proportional) fictitious Neumann condition. We next deduce in detail the decomposed form of the lower bound optimisation problem.

As described in Section 1, equation $\overline{\mathbf{A}}^{e q 1} \sigma+F^{e q 1}=0$ in (4) is related to the equilibrium constraint, which can be decomposed into two separate equations as follows:

$$
\begin{aligned}
& \overline{\mathbf{A}}^{e q 1,1} \sigma^{1}+\lambda F^{e q 1,1}=\mathbf{0}, \\
& \overline{\mathbf{A}}^{e q 1,2} \sigma^{2}+\lambda F^{e q 1,2}=\mathbf{0} .
\end{aligned}
$$

When the vector $\sigma$ is split into two vectors $\sigma^{1}$ and $\sigma^{2}$, the domain of the problem is also split into two parts with a common boundary that couples some of the two set 


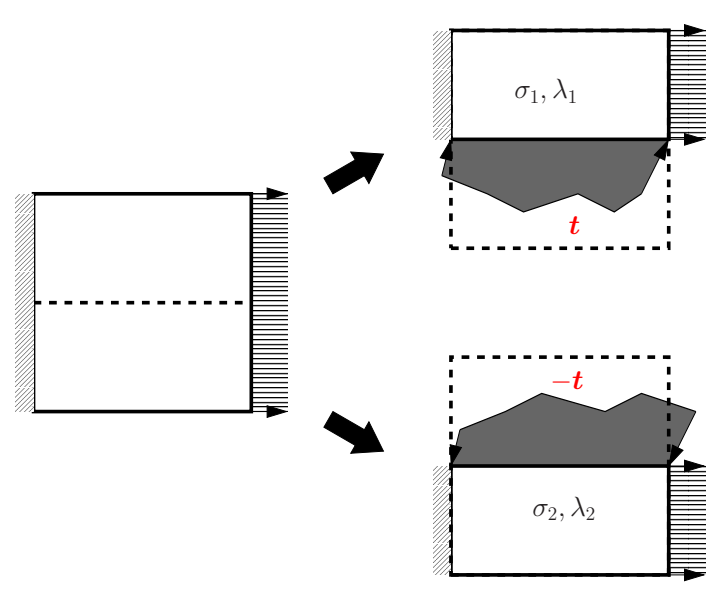

Fig. 8 Decomposition of the global domain of a limit analysis problem (left) into two domains, with the fictitious Neumann boundary shaded (right).

of variables. This means that the vectors $\sigma^{1}$ and $\sigma^{2}$ may be decomposed into two vectors $\sigma^{1}=\left(\sigma^{1,1}, \sigma^{1,2}\right)$ and $\sigma^{2}=\left(\sigma^{2,1}, \sigma^{2,2}\right)$ such that the vectors $\sigma^{1,1}$ and $\sigma^{2,2}$ are coupled through the inter-element equilibrium constraints as follows:

$$
\begin{aligned}
& \overline{\mathbf{A}}^{e q 2,1} \sigma^{1}=0 \\
& \overline{\mathbf{A}}^{e q 2,2} \sigma^{2}=0 \\
& \overline{\mathbf{B}}^{e q 2,1} \sigma^{1}+\overline{\mathbf{B}}^{e q 2,2} \sigma^{2}=0 .
\end{aligned}
$$

We note that the last equation in (22) is a complicating constraint. The equation $\overline{\mathbf{A}}^{3} \sigma+\lambda F^{e q 3}=0$ is separable and can be in turn decomposed as,

$$
\begin{aligned}
& \overline{\mathbf{A}}^{e q 3,1} \sigma^{1}+\lambda F^{e q 3,1}=0 \\
& \overline{\mathbf{A}}^{e q 3,2} \sigma^{2}+\lambda F^{e q 3,2}=0
\end{aligned}
$$

Consequently, after applying the linear transformation to variable $\sigma^{i}$, we can rewrite the optimisation problem in (6) as,

$$
\begin{aligned}
& \min _{x_{1}, x_{2}, \lambda}-\lambda \\
& \mathbf{A}^{e q 1, i} x^{i}+\lambda F^{e q 1, i}=b^{e q 1, i}, \quad i=1,2 \\
& \mathbf{A}^{e q 2, i} x^{i}=0, \quad i=1,2 \\
& \mathbf{A}^{e q 3, i} x^{i}+\lambda F^{e q 3, i}=b^{e q 3, i}, \quad i=1,2 \\
& \mathbf{B}^{e q 2,1} x^{1}+\mathbf{B}^{e q 2,2} x^{2}=0 \\
& x^{1} \in K_{1}, x^{2} \in K_{2} ; \lambda \text { free. }
\end{aligned}
$$


The last equation in the previous optimisation problem (24) is a complicating constraint of the local variables $x_{1}$ and $x_{2}$, while the variable $\lambda$ can be regarded as a global variable. In order to decompose the problem in (24) we first introduce a variable $t$ such that

$$
\mathbf{B}^{e q 2,1} x^{1}=t,
$$

Then we can rewrite the optimisation problem in the following form:

$$
\begin{aligned}
& \min _{t, x_{i}, \lambda}-\lambda \\
& \quad \mathbf{A}^{e q 1, i} x^{i}+\lambda F^{e q 1, i}=b^{e q 1, i} \\
& \quad \mathbf{A}^{e q 2, i} x^{i}=0 \\
& \mathbf{A}^{e q 3, i} x^{i}+\lambda F^{e q 3, i}=0 \\
& \mathbf{B}^{e q 2, i} x^{i}=(-1)^{i+1} t \\
& x_{i} \in K_{i}, t, \lambda \text { free },(i=1,2)
\end{aligned}
$$

Note that since the complicating constraint in optimisation problem (25) is built through the common boundary, the coupling constraint can be interpreted as fictitious Newman condition for each sub-domain.

By introducing new variables $t_{i},(i=1,2)$ we can rewrite our problem in the following form:

$$
\begin{aligned}
& \min \left(-\frac{\lambda_{1}}{2}\right)+\left(-\frac{\lambda_{2}}{2}\right) \\
& \mathbf{A}^{e q 1, i} x^{i}+\lambda_{i} F^{e q 1, i}=b^{e q 1 . i} \\
& \mathbf{A}^{e q 2, i} x^{i}=0 \\
& \mathbf{A}^{e q 3, i} x^{i}+\lambda_{i} F^{e q 3, i}=b^{e q 3, i} \\
& \mathbf{B}^{e q 2, i} x^{i}=(-1)^{i+1} t_{i} \\
& t_{1}=t_{2} \\
& \lambda_{1}=\lambda_{2} .
\end{aligned}
$$

Let $C_{i},(i=1,2)$ be local constraints that are defined as follows:

$$
C_{i}=\left\{\begin{array}{c}
\mathbf{A}^{e q 1, i} x^{i}+\lambda_{i} F^{e q 1, i}=b^{e q 1, i} \\
\mathbf{A}^{e q 2, i} x^{i}=0 \\
\left\{t_{i}, x^{i}, \lambda^{i}\right\} \mid \mathbf{A}^{e q 3, i} x^{i}+\lambda_{i} F^{e q 3, i}=b^{e q 3, i} \\
\mathbf{B}^{e q 2, i} x^{i}=t_{i} \\
x^{i} \in K_{i}, t_{i}, \lambda_{i} \text { free. }
\end{array}\right\}
$$

In the above problem, $t_{1}, \lambda_{1}, t_{2}, \lambda_{2}$ are public variables and $x^{1}, x^{2}$ are private variables. Let us collect all the public variables together into one variable $y=$ $\left(t_{1}, \lambda_{1}, t_{2}, \lambda_{2}\right)=\left(y_{1}, y_{2}\right)$ where $y_{1}=\left(t_{1}, \lambda_{1}\right), y_{2}=\left(t_{2}, \lambda_{2}\right)$. If we introduce a vector $z$ that gives the common values of the public variables, then we can express the 
coupling constraints as $y=\mathbf{E} z$ where $\mathbf{E}$ is a matrix with components equal to zero or one, that is:

$$
z=\left\{\begin{array}{l}
t \\
\lambda
\end{array}\right\}, y_{i}=\mathbf{E}_{i} z \quad \text { where } \quad \mathbf{E}_{i}=\left[\begin{array}{ll}
\mathbf{I} & 0 \\
0 & 1
\end{array}\right], y=\mathbf{E} z \quad \text { where } \quad \mathbf{E}=\left[\begin{array}{l}
\mathbf{E}_{1} \\
\mathbf{E}_{2}
\end{array}\right] .
$$

Let us set $f_{i}\left(x_{i}, y_{i}\right)=f\left(x_{i}, t_{i}, \lambda_{i}\right)=-\frac{\lambda_{i}}{2}(i=1,2)$. Then our problem has the followings form:

$$
\begin{aligned}
\min _{x, y, z} & f_{1}\left(x_{1}, y_{1}\right)+f_{2}\left(x_{2}, y_{2}\right) \\
& \left(x_{1}, y_{1}\right) \in C_{1}, \quad\left(x_{2}, y_{2}\right) \in C_{2} \\
& y_{i}=\mathbf{E}_{i} z \quad i=1,2 \\
& x=\left(x_{1}, x_{2}\right), y=\left(y_{1}, y_{2}\right), y_{i}=\left(t_{i}, \lambda_{i}\right),(i=1,2),
\end{aligned}
$$

with variables $x_{i}, y_{i}$, and $z$. We then have a problem that is separable for fixed values of $z$.

\subsubsection{Primal decomposition of LB problem}

In primal decomposition, at each iteration we fix the vector $z$ and we fix the public variables as $y_{i}=\mathbf{E}_{i} z$. The problem is now separable. Each sub-problem can separately find optimal values for its local variables $x_{i}$. Let us denote $q_{i}\left(y_{i}\right)=q_{i}(\mathbf{E} z)$ the optimal value of the sub-problem

$$
\begin{aligned}
q_{i}\left(y_{i}\right)=\min _{x_{i}} f_{i}\left(x_{i}, y_{i}\right) & \\
& \left(x_{i}, y_{i}\right) \in C_{i},(i=1,2),
\end{aligned}
$$

with variable $x_{i}$, as a function of $y_{i}$. The original problem (26) is equivalent to the primal master problem

$$
\min _{z} q(z)=q_{1}\left(\mathbf{E}_{1} z\right)+q_{2}\left(\mathbf{E}_{2} z\right)
$$

with variable $z$. In order to find a sub-gradient of $q$, denoted by $g$, we find $g_{i} \in \partial q_{i}\left(y_{i}\right)$ (which can be done separately), and then compute $g$ as,

$$
g=\mathbf{E}_{1}^{T} g_{1}+\mathbf{E}_{2}^{T} g_{2} .
$$

\subsubsection{Dual decomposition of LB problem}

We form the partial Lagrangian of problem (26), 


$$
\begin{aligned}
L(x, y, z, v) & =f_{1}\left(x_{1}, y_{1}\right)+f_{2}\left(x_{2}, y_{2}\right)+v^{T}(-y+\mathbf{E} z) \\
& =\left(f_{1}\left(x_{1}, y_{1}\right)-v_{1}^{T} y_{1}\right)+\left(f_{2}\left(x_{2}, y_{2}\right)-v_{2}^{T} y_{2}\right)+v^{T} \mathbf{E} z,
\end{aligned}
$$

where $v$ is the Lagrangian multiplier associated with $y=\mathbf{E} z$. To find the dual function, we first minimize over $z$, which results in the condition $\mathbf{E}^{T} v=0$. In other words

$$
\begin{gathered}
q(v)=q\left(v_{1}, v_{2}\right)=\min _{x_{1}, x_{2}, y_{1}, y_{2}} \min _{z}\left(f_{1}\left(x_{1}, y_{1}\right)-v_{1}^{T} y_{1}\right)+\left(f_{2}\left(x_{2}, y_{2}\right)-v_{2}^{T} y_{2}\right)+v^{T} \mathbf{E} z \\
\left(x_{1}, y_{i}\right) \in C_{i},(i=1,2),
\end{gathered}
$$

then

$$
\begin{gathered}
q(v)=q\left(v_{1}, v_{2}\right)=\min _{x_{1}, x_{2}, y_{1}, y_{2}}\left(f_{1}\left(x_{1}, y_{1}\right)-v_{1}^{T} y_{1}\right)+\left(f_{2}\left(x_{2}, y_{2}\right)-v_{2}^{T} y_{2}\right) \\
\mathbf{E}^{T} v=0 \\
\left(x_{i}, y_{i}\right) \in C_{i},(i=1,2) .
\end{gathered}
$$

We define $q_{i}\left(v_{i}\right),(i=1,2)$ as the optimal value of the sub-problems, $(i=1,2)$,

$$
\begin{gathered}
q_{i}\left(v_{i}\right)=\min _{x_{i}, y_{i}}\left(f_{i}\left(x_{i}, y_{i}\right)-v_{i}^{T} y_{i}\right) \\
\left(x_{i}, y_{i}\right) \in C_{i},
\end{gathered}
$$

as a function of $v_{i}$. A sub-gradient of $q_{i}$ at $v_{i}$ is just $-y_{i}$, an optimal value of $y_{i}$ in the sub-problem (27). Therefore the dual of the original problem (26) is

$$
\begin{aligned}
\max q(v) & =q_{1}\left(v_{1}\right)+q_{2}\left(v_{2}\right) \\
\mathbf{E}^{T} v & =0,
\end{aligned}
$$

with variable $v$. We can solve this dual decomposition master problem using a projected sub-gradient method. The projection onto the feasible set $\left\{v \mid \mathbf{E}^{T} v=0\right\}$, is given by the following operator:

$$
\mathbf{I}-\mathbf{E}\left(\mathbf{E}^{T} \mathbf{E}\right)^{-1} \mathbf{E}^{T} .
$$

\section{Numerical results}

\subsection{Bearing capacity of anchors}

The extensions described in Section 2 have been employed to test the pull out capacity of multi-belled anchors. The linearity of the limit tension with respect to the number of bells has been verified.

Five different anchor/soil conditions have been employed: rough (same properties as the soil), smooth (no resistance to shear), no tension condition, rough con- 
dition with no tension, and smooth condition with no-tension. Although the mechanisms do not significantly depend on these conditions, the pull out capacity does, and has been shown to be much larger for rough conditions. On the other hand, while for clay materials (zero internal friction angle, but non-zero cohesion) the failure mechanism is localised around the anchor (see Figure 9a), in other sand materials the slide-lines propagate up to the soil surface (see Figure 9b). The computed limiting height agrees satisfactorily with experimental results and other numerical models that use incremental plasticity [16].

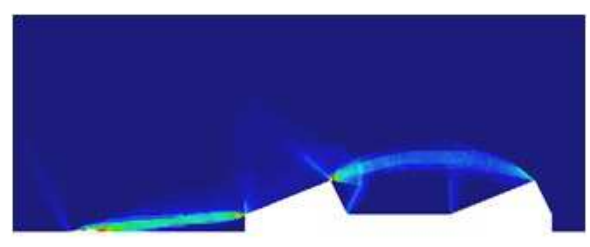

(a)

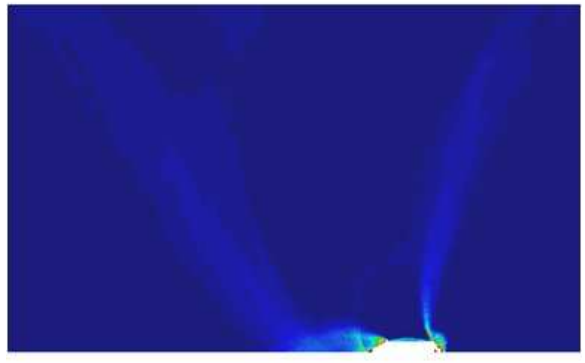

(b)

Fig. 9 Dissipation energy of double bell anchor. (a): Clay soil, with sliding conditions and no tension at the anchor-soil interface. (b): sand soil with internal friction angle $\phi=20^{\circ}$, and rough interface conditions with no tension.

\subsection{Retaining walls}

The maximum height of a simply supported and anchored retaining wall has been computed using the techniques explained in Section 2. Figure 10 shows the dissipation power of an anchored wall, with a zoom on the region surrounding the anchor. For an anchor length equal to the height of the wall, the collapse mechanism suround the whole wall-anchor system, while for longer anchors, the mechanism tends to be localised around the anchor. Figure 11 shows the mesh obtained after 4 successive iterations. As it can be observed, the smaller elements localise in the regions with higher dissipation power and at the slidelines.

The tests have been run for different ratios of $d / h$ where $h=$ free wall height, and $d=$ total height of the wall. The collapse of the wall was obtained for a certain factor $\lambda$ of the gravity acceleration. As the ratio $h / d$ is increased, the value of $\lambda$ was decreasing. The limiting free height is the value for which $\lambda=1$. The plots in Figure 12 show the evolution of $\lambda$, and indicate the limiting ratios for different wall conditions and admissibility conditions of the wall-soil and anchor-soil interfaces. These values agree with some experimental values published in [19]. 

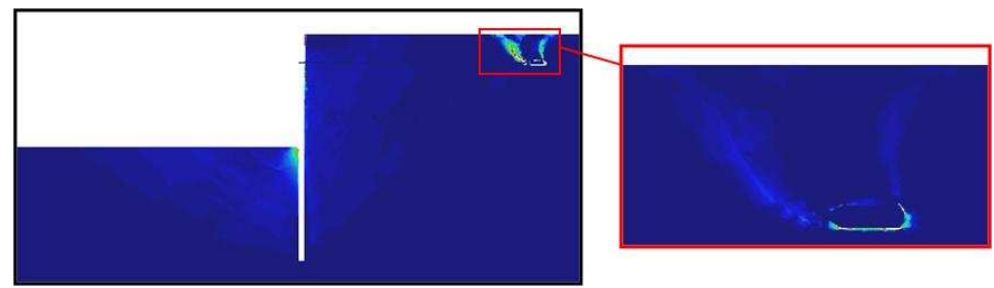

Fig. 10 Contour plot of the dissipation power on an anchored wall.Left: domain without the wall. Right: zoom on the domain surrounding the anchor. Interface soil-acnhor and soil-wall conditions are rough with no tension.

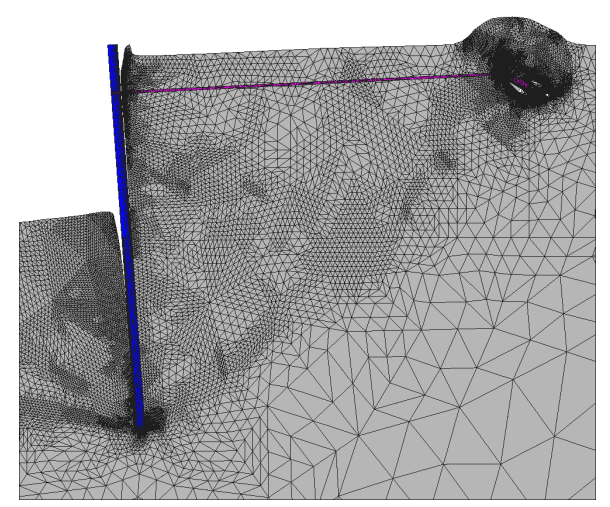

Fig. 11 Upper bound velocities of anchored wall with the resulting mesh after 4 meshing strategies.

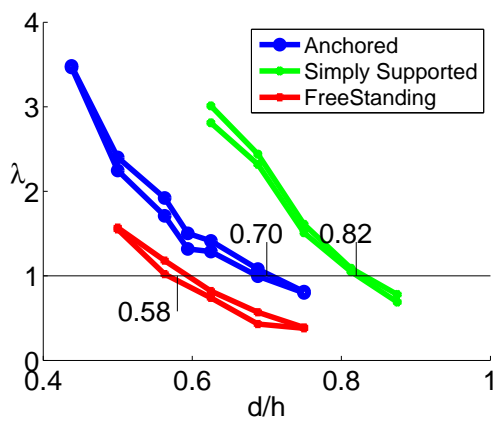

(a)

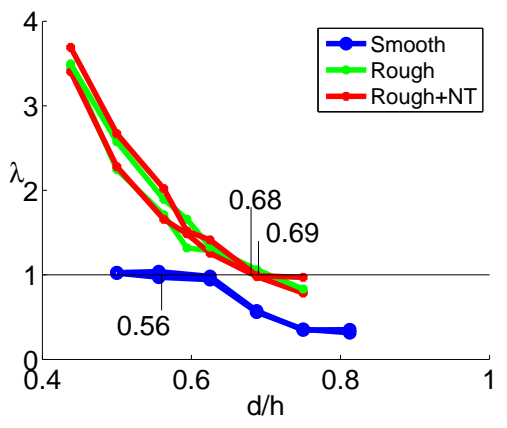

(b)

Fig. 12 (a) Upper and lower bound of the bearing capacity of a retaining wall with a rough interface with no traction. (b) Bounds for the anchored wall with different anchor-soil conditions. Horizontal axis corresponds to the ratio $d / h$, while the values in the vertical axis corresponds to the factor multiplying the gravity acceleration at collapse.

\subsection{Decomposition techniques}

We have applied the dual decomposition technique described in Section 3.2.3 with dynamic step size [3] to the LB problem using a mesh with 128 elements and 
1537 primal variables (see Figure 13a). The domain is subjected to a horizontal traction field at the right boundary, and to a fully homogeneous Dirichlet conditions on its left boundary.

Figure 13b shows the evolution of the upper and lower bounds of the LB optimization problem, which after successive iteration converges to the exact value $\lambda^{*}=\lambda^{L B}=2$. The algorithm converges to tolerance of $10^{-3}$ for the relative difference of upper and lower bound, the number of master iterations are between 10 and 15.

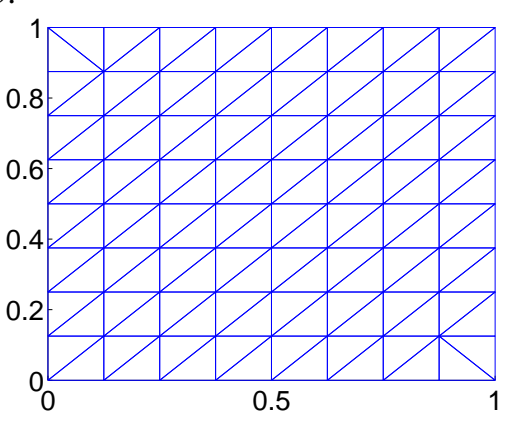

(a)

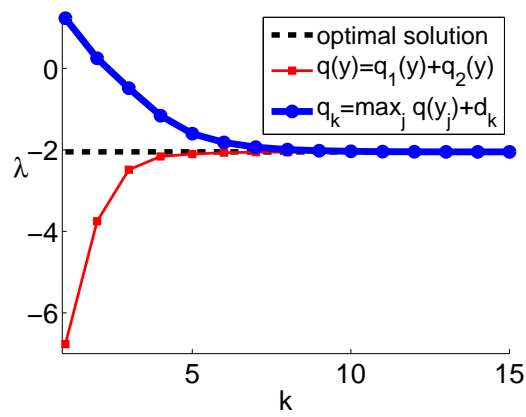

(b)

Fig. 13 a) Domain and mesh employed in the decomposition analysis of the lower bound problem. The domain is subjected to a homogeneous Dirichlet conditions (zero velocities) at the left boundary, and a horizontal tensile traction at the right boundary. b) Evolution of the upper and lower bound of the optimal solution of the LB limit analysis problem when using a variable step-size in the dual decomposition method.

\section{Conclusions}

We have presented some recent extension of the lower and upper bound formulation of limit analysis for problems with specific frictional interfaces, duplicated edges and joints. Such extensions are motivated by the limit analysis of practical problems with anchors and other structural elements.

We have also described how the optimisation problems that current computational limit analysis encounters may be decomposed for its eventual parallelisation. Although only simple domains problems with limited number of elements have been analysed, the methodology presented here is general and may be applied for more general problems. This generalisation, and other techniques are currently under investigation. 


\section{References}

1. MOSEK ApS. The MOSEK optimization tools version 3.2 (Revision 8). User's Manual and Reference, 2005. Avail. http://www.mosek.com.

2. J F Bendres. Partitioning procedures for solving mixed-variables programming problems. Numer. Math., 4:238-252, 1962.

3. D P Bertsekas. Convex analysis and optimization. Athena Scientific, 3rd edition, 2003.

4. S Boyd and L Vandenberghe. Convex Optimization. Cambridge Univ. Press, 2004.

5. S Boyd, L Xiao, A Mutapcic, and J Mattingley. Notes on decomposition methods. Technical report, Stanford University, 2007. Notes of EE364B course.

6. E Christiansen. Handbook of Numerical Analysis, vol IV, volume IV, chapter II, Limit Analysis of Collapse States, pages 193-312. North Holland Amsterdam, 1996.

7. H Ciria, J Peraire, and J Bonet. Mesh adaptive computation of upper and lower bounds in limit analysis. Int. J. Num. Meth. Engng., 75:899-944, 2008.

8. AJ Conejo, E Castillo, R Mínguez, and R García-Bertrand. Decomposition Techniques in Mathematical Programming. Springer, The Netherlands, 2006.

9. A M Geoffrion. Generalized Benders Decomposition. J. Optim. Th. Appl., 10(4):238-252, 1972.

10. con J Salen'De l'Élasto-plasticité au Calcul à la rupture. Les Éditions de l'Ècole Polytechnique, 2002.

11. Z Kammoun, F Pastor, H Smaoui, and J Pastor. Large static problem in numerical limit analysis: A decomposition approach. Int. J. Num. Anal. Meth. Geomech., 34:1960-1980, 2010.

12. I Kaneko. A decomposition procedure for large-scale optimum plastic design problems. Int. J. Num. Meth. Engng., 19:873-889, 1983.

13. A V Lyamin, S W Sloan, K Krabbenhøft, and M Hjiaj. Lower bound limit analysis with adaptive remeshing. Int. J. Num. Meth. Engng., 63:1961-1974, 2005.

14. J J Muñoz, J Bonet, A Huerta, and J Peraire. Upper and lower bounds in limit analysis: adaptive meshing strategies and discontinuous loading. Int. J. Num. Meth. Engng., 77:471$501,2009$.

15. J J Muñoz, J Bonet, A Huerta, and J Peraire. A note on upper bound formulations in limit analysis. Int. J. Num. Meth. Engng., 91(8):896-908, 2012.

16. J J Muñoz, A Lyamin, and A Huerta. Stability of anchored sheet wall in cohesive-frictional soils by FE limit analysis. Int. J. Num. Anal. Meth. Geomech., 2012. In press. DOI: 10.1002/nag.2090.

17. J F Sturm. Using SeDuMi 1.02, a MATLAB toolbox for optimization over symmetric cones. Optimization Methods and Software, 11-12:625-653, 1999. Version 1.05 available from http://http://sedumi.ie.lehigh.edu.

18. R H Tütüncü, KC Toh, and MJ Todd. Solving semidefinite-quadratic-linear programs using SDPT3. Mathem. Progr. Ser. B, 95:189-217, 2003. Avail. http://www.math.nus.edu.sg/ mattohkc/sdpt3.html.

19. K C Vossoughi. Étude numérique du comportement des ouvrages de soutènement à la rupture. $\mathrm{PhD}$ thesis, Ecole Centrale Paris, Paris, France, 2001. 